\title{
OPTIMAL DISCRETE-TIME MAGNETIC ATTITUDE CONTROL OF SATELLITES
}

\author{
M. Lovera* and A. Varga ${ }^{* *}$ \\ * Dipartimento di Elettronica e Informazione \\ Politecnico di Milano \\ P.za Leonardo da Vinci 32, 20133 Milano, Italy \\ Phone: + 3902 23993592; Fax: +3902 23993412 \\ Email: lovera@elet.polimi.it \\ ** German Aerospace Center, DLR-Oberpfaffenhofen \\ Institute of Robotics and Mechatronics \\ D-82234 Wessling, Germany \\ Email: andras.varga@dlr.de
}

\begin{abstract}
The problem of designing discrete-time attitude controllers for magnetically actuated spacecraft is considered. Several methods are discussed and a novel approach to the tuning of "projection based" controllers is proposed relying on periodic optimal output feedback control techniques. The main advantages of the proposed method are discussed and illustrated in a simulation study. Copyright ${ }^{\circledR} 2005$ IFAC
\end{abstract}

Keywords: Satellite control, attitude control, optimal control, discrete-time systems, periodic systems.

\section{INTRODUCTION}

Electromagnetic actuators are a particularly effective and reliable technology for the attitude control of small satellites. Such actuators operate on the basis of the interaction between the magnetic field generated by a set of three orthogonal, current-driven coils and the magnetic field of the Earth and therefore provide a very simple solution to the problem of generating torques on board of a satellite. More precisely, magnetic torquers can be used either as main actuators for attitude control in momentum biased or gravity gradient attitude control architectures or as secondary actuators for momentum management tasks in zero momentum reaction wheel based configurations.

The use of electromagnetic actuators, however, gives rise to a number of difficulties when it comes to control system design. The torques which can be generated in this way are instantaneously constrained to lie in the plane orthogonal to the local magnetic field vector. In spite of this constraint, controllability of the attitude dynamics is ensured for a wide range of orbit altitudes and inclinations thanks to the variability of the geomagnetic field. However, the control designer is faced with the task of working out a suitable timevarying control law to deal with such effects. In recent years, a considerable effort has been devoted to the analysis of this control problem (see, e.g., (Arduini and Baiocco, 1997; Wisniewski and Blanke, 1999)); in particular, as the variability of the geomagnetic field is almost time-periodic, most of the recent work on the linear attitude control problem has focused on the use of optimal and robust periodic control theory for the design of state and output feedback regulators (Pittelkau, 1993; Varga and Pieters, 1998; Wisniewski and Markley, 1999; Lovera et al., 2002; Psiaki, 2001; Lovera, 2001). See also (Silani and Lovera, 2005) for a recent survey on this subject. However, in spite of the extensive activity, the development of a design technique leading to a simple, easily implementable, yet efficient controller remains an open problem. 
The aim of this paper is to propose and compare a number of different approaches to the design of digital attitude controllers for spacecraft equipped with magnetic actuators, with specific emphasis on practical aspects associated with their on-board implementation. In particular, the results obtained using periodic optimal state feedback and predictive state feedback control are compared with the ones provided by a novel approach to the tuning of a classical fixed structure controller for magnetic attitude control known as the "projection based" controller.

It is important to note that the design problems associated with periodic optimisation techniques pose a significant challenge from the numerical point of view (unstable open loop dynamics and very large period) and could be only solved by using reliable numerical methods as those implemented in the Periodic Systems Toolbox for Matlab (Varga, 2005b).

\section{SPACECRAFT MODEL}

For the purpose of the present analysis, the following coordinate systems are adopted:

- Yaw-Roll-Pitch (orbital) axes. The origin of these axes is in the satellite centre of mass. The $\mathrm{X}$-axis is defined as the vector pointing towards the Earth's centre and positive in the same direction. The Y-axis points in the direction of the orbital velocity vector. The $\mathrm{Z}$-axis is normal to the satellite orbit plane and completes the righthanded orthogonal triad.

- Satellite body axes. The origin of these axes is in the satellite centre of mass; the axes are assumed to coincide with the body's principal inertia axes.

As is well known (Wertz, 1978), the attitude matrix $\mathbf{A}(q)$ with respect to the orbital axes can be expressed as a function of the (unit norm) quaternion vector $q=\left[\begin{array}{llll}q_{1} & q_{2} & q_{3} & q_{4}\end{array}\right]^{T}:=\left[\begin{array}{ll}q_{R}^{T} & q_{4}\end{array}\right]^{T} \in \mathbb{R}^{4}$ and the time evolution of the attitude parameters as a function of the angular rate $\omega_{r}$ of the spacecraft relative to a desired reference frame can be described by the kinematic equations

$$
\dot{q}=\frac{1}{2} W\left(\omega_{r}\right) q
$$

where $W\left(\omega_{r}\right)$ depends on the components of $\omega_{r}$ as

$$
W\left(\omega_{r}\right)=\left[\begin{array}{cccc}
0 & \omega_{r z} & -\omega_{r y} & \omega_{r x} \\
-\omega_{r z} & 0 & \omega_{r x} & \omega_{r y} \\
\omega_{r y} & -\omega_{r x} & 0 & \omega_{r z} \\
-\omega_{r x} & -\omega_{r y} & -\omega_{r z} & 0
\end{array}\right]
$$

For Earth pointing spacecraft (on circular orbits) one spontaneously considers the orbital coordinate frame as an attitude reference, so that $\omega_{r}=\omega-$ $\mathbf{A}(q)\left[\begin{array}{lll}0 & 0 & -\Omega_{0}\end{array}\right]^{T}$, where $\Omega_{0}$ is the orbital angular rate and $\omega$ is angular rate of the body reference frame.
The equations of angular dynamics (Wertz, 1978; Sidi, 1997) can be expressed in vector form as

$$
\frac{d h}{d t}=T
$$

where $h \in \mathbb{R}^{3}$ is the overall angular momentum of the spacecraft and $T$ is the sum of the external torques (disturbance and control ones) acting on the satellite. The derivative of $h$ is here expressed in an inertial reference frame. Considering instead the body reference frame, rotating with angular rate $\omega$, the Euler's equations become

$$
\dot{h}=-\omega \times h+T \text {. }
$$

For a rigid spacecraft $h=I \omega$, where $I \in \mathbb{R}^{3 \times 3}$ is the spacecraft inertia matrix expressed in body frame, so Euler's equations reduce to the well known form

$$
I \dot{\omega}=-\omega \times I \omega+T=S(\omega) I \omega+T,
$$

where $S(\omega)$ is given by

$$
S(\omega)=\left[\begin{array}{ccc}
0 & \omega_{z} & -\omega_{y} \\
-\omega_{z} & 0 & \omega_{x} \\
\omega_{y} & -\omega_{x} & 0
\end{array}\right] .
$$

In this paper we consider the case of a momentum biased satellite, i.e., a spacecraft equipped with one momentum wheel, aligned with the $\mathrm{z}$ body axis. The wheel is characterised by a moment of inertia matrix $J$ and a velocity vector $\Omega$ relative to the body frame, and therefore an angular momentum $h_{w}=\left[\begin{array}{lll}0 & 0 & J\end{array}\right]^{T}$. We have that $h=I \omega+h_{w}$ and therefore (3) becomes

$$
I \dot{\omega}+\dot{h}_{w}=S(\omega)\left[I \omega+h_{w}\right]+T .
$$

It will be assumed in the following that the angular velocity of the momentum wheel is kept constant to a nominal value $\bar{\Omega}$, so that we have $\dot{h}_{w}=0$ and $h_{w}$ constant in equation (5). Note that the external torque $T$ appearing in equation (2) can be decomposed into the sum of three terms, namely the gravity gradient torque $T_{g g}$ (which will be included in the linearized dynamics), the magnetic control torque $T_{c, m a g}$ (i.e., the control torque generated by the magnetic coils) and the sum of disturbance torques $T_{c, \text { dist }}$.

In particular, disturbance torques due to gravity gradient are taken into account in the spacecraft dynamics as they have an important effect in determining the stability characteristics of the equilibria of relative motion for Earth pointing spacecraft (see (Hughes, 1986)). Such torques are given by

$$
T_{g g}=3 \Omega_{0}^{2} z \times I z
$$

where $z \in \mathbb{R}^{3}$ is the local zenith direction in body axes. For example, in the case of an Earth pointing satellite one has

$$
z=\mathbf{A}(q)\left[\begin{array}{lll}
-1 & 0 & 0
\end{array}\right]^{T} .
$$

Finally, the control torques generated by the magnetic coils are given by the expression:

$$
T_{c, \text { mag }}=m_{c} \times b=S(b) m_{c}
$$


with $S(\cdot)$ as defined in (4) and where the vector $b \in \mathbb{R}^{3}$ is formed with the components of geomagnetic field vector with respect to the body frame, and $m_{c} \in \mathbb{R}^{3}$ is the vector of the coils' magnetic dipoles. According to equation (8) the control torque of the magnetically controlled satellite is constrained to be orthogonal to the local direction of the geomagnetic field vector. This also implies that the component of the magnetic moment in the direction parallel to the local geomagnetic field has no influence on the satellite motion. Note that even if $S(b)$ is structurally singular, "average" controllability (i.e., controllability in the time-varying sense) is guaranteed for most orbits of practical interest thanks to the variability of $b$.

In this study we consider a spacecraft such that $I=$ $\operatorname{diag}\left[\begin{array}{lll}I_{x x} & I_{y y} & I_{z z}\end{array}\right]$, equipped with a single momentum/reaction wheel aligned with the body $z$ axis, with moment of inertia $J$ and angular velocity $\Omega$ relative to the body frame. For this spacecraft configuration the aim of the attitude control scheme is to maintain the spacecraft (body axes) aligned with the orbital axes, while exploiting the gyroscopic effect due to the momentum wheel. In the following we will derive linearised dynamic models for the formulation of this control problem.

Define the state vector $x_{c}=\left[\delta q_{R}^{T} \delta \omega^{T}\right]^{T}$ formed with small displacements of the vector part $q_{R}$ of the attitude quaternion with respect to the orbital axes from the nominal values $\bar{q}_{R}=\left[\begin{array}{lll}0 & 0 & 0\end{array}\right]^{T}$ and small deviations of the body rates from the nominal values $\bar{\omega}_{x}=\bar{\omega}_{y}=0, \bar{\omega}_{z}=-\Omega_{0}$. Then the attitude dynamics can be linearized and the local linear dynamics for the attitude can be defined as

$$
\dot{x}_{c}(t)=A_{c} x_{c}(t)+B_{c T}\left[T_{c, \text { mag }}(t)+T_{c, \text { dist }}(t)\right]
$$

or

$$
\dot{x}_{c}(t)=A_{c} x_{c}(t)+B_{c m}(t) m_{c}(t)+B_{c T} T_{c, d i s t}(t)(10)
$$

where

$$
\begin{aligned}
& A_{c}=\left[\begin{array}{cccccc}
0 & -\Omega_{0} & 0 & 0.5 & 0 & 0 \\
\Omega_{0} & 0 & 0 & 0 & 0.5 & 0 \\
0 & 0 & 0 & 0 & 0 & 0.5 \\
0 & 0 & 0 & 0 & W_{x} & 0 \\
0 & -6 k_{y} \Omega_{0}^{2} & 0 & W_{y} & 0 & 0 \\
0 & 0 & +6 k_{z} \Omega_{0}^{2} & 0 & 0 & 0
\end{array}\right] \\
& B_{c T}=\left[\begin{array}{ccc}
0 & 0 & 0 \\
0 & 0 & 0 \\
0 & 0 & 0 \\
I_{x x}^{-1} & 0 & 0 \\
0 & I_{y y}^{-1} & 0 \\
0 & 0 & I_{z z}^{-1}
\end{array}\right], \quad B_{c m}(t)=B_{c T} S(b(t))
\end{aligned}
$$

and $k_{x}=\frac{I_{y y}-I_{z z}}{I_{x x}}, k_{y}=\frac{I_{z z}-I_{x x}}{I_{y y}}, k_{z}=\frac{I_{x x}-I_{y y}}{I_{z z}}$, $W_{x}=-k_{x} \Omega_{0}-k_{w x} \bar{\Omega}, W_{y}=-k_{y} \Omega_{0}+k_{w y} \bar{\Omega}$, $k_{w x}=\frac{J}{I_{x x}}, k_{w y}=\frac{J}{I_{y y}}$. Here, $\bar{\Omega}$ is the nominal wheel speed.

Note that two different control matrices $B_{c T}$ and $B_{c m}(t)$ have been defined, in order to handle prob- lem formulations in which either magnetic torques $\left(T_{c, \text { mag }}\right)$ or magnetic dipoles $\left(m_{c}\right)$ are used as control variables, respectively. Therefore, while $A_{c}$ is constant, the control matrix $B_{c m}(t)$ corresponding to the control input $m_{c}$ turns out to be time-varying (and approximately time-periodic with period $2 \pi / \Omega_{0}$ ) because of the dependence on $b(t)$.

Finally, since we are concerned with a discrete-time design problem, suitable discrete-time equivalents of (9) and (10) have been derived, in the forms

$$
\begin{aligned}
& x(k+1)=A x(k)+B_{T}\left[T_{\text {mag }}(k)+T_{\text {dist }}(k)\right] \\
& x(k+1)=A x(k)+B_{m}(k) m(k)+B_{T} T_{\text {dist }}(k)(12)
\end{aligned}
$$

respectively, where for a sampling-time of $\Delta=$ $2 \pi /\left(N \Omega_{0}\right)$ ( $N$ is the discrete-time period) we have: $A:=\exp \left(A_{c} \Delta\right), B_{T}:=\int_{0}^{\Delta} e^{A_{c}(\Delta-\tau)} B_{c T} d \tau$, $B_{m}(k):=\int_{k \Delta}^{(k+1) \Delta} e^{A_{c}[(k+1) \Delta-\tau]} B_{c m}(\tau) d \tau, x(k):=$ $x_{c}(k \Delta), T_{\text {mag }}(k):=T_{c, \text { mag }}(k \Delta), T_{\text {dist }}(k):=$ $T_{c, d i s t}(k \Delta)$, and $m(k):=m_{c}(k \Delta)$.

\section{CONTROLLER DESIGN}

\subsection{Periodic optimal state feedback controller}

Consider the system (12) and let $u(k)=m(k)$. Minimizing the linear-quadratic (LQ) criterion

$$
J=\sum_{k=0}^{\infty}\left[x(k)^{T} Q x(k)+u(k)^{T} R u(k)\right]
$$

where $Q \geq 0, R>0$ are symmetric matrices, is an attractive method to determine stabilizing periodic state feedback controllers of the form

$$
u(k)=F(k) x(k)
$$

The optimal $N$-periodic state-feedback matrix $F(k)$ minimizing the performance index (13) is given by

$$
\begin{aligned}
F(k)=-\left(R+B_{m}^{T}(k) X(k+1) B_{m}(k)\right)^{-1} \\
\cdot B_{m}^{T}(k) X(k+1) A
\end{aligned}
$$

where the $N$-periodic symmetric positive semi-definite matrix $X(k)$ satisfies the reverse discrete-time periodic Riccati equation

$$
\begin{gathered}
X(k)=Q+A^{T} X(k+1) A \\
-A^{T} X(k+1) B_{m}(k)\left(R+B_{m}^{T}(k) X(k+1) B_{m}(k)\right)^{-1} \\
\cdot B_{m}^{T}(k) X(k+1) A
\end{gathered}
$$

This periodic Riccati equation can be solved using the algorithm proposed in (Varga, 2005a) implemented in the Periodic Systems Toolbox (Varga, 2005b).

The optimal periodic LQ approach has the obvious advantage of providing a controller with a very good level of performance. This is the reason why optimal periodic control has been extensively studied as a viable approach to this problem, in a number of different settings and formulations: continuous-time in (Pittelkau, 1993; Wisniewski and Markley, 1999; 
Lovera et al., 2002; Lovera, 2001) and discrete-time in (Wisniewski and Stoustrup, 2002). The issues associated with the implementation of optimal periodic controllers, however, make their actual application in real satellite missions not very likely: the storage requirements for a fully time-periodic gain are indeed a critical problem. While these issues motivate the interest in alternative approaches to this design problem, the performance level provided by the optimal periodic LQ controller can be taken as a reference for all other approaches.

\subsection{Predictive magnetic controller}

Consider now system (11), and let $u(k)=T_{\text {mag }}(k)$. The optimal $j$-ahead prediction of the state for this system is given by (Camacho and Bordons, 1998):

$$
\hat{x}(k+j \mid k)=A^{j} x(k)+\sum_{i=0}^{j-1} A^{j-i-1} B_{T} u(k+i)
$$

Let us consider a set of $N_{c}$ (control horizon) $j$-ahead predictions

$$
\begin{aligned}
& \mathbf{x}:=\left[\begin{array}{c}
\hat{x}(k+1 \mid k) \\
\hat{x}(k+2 \mid k) \\
\vdots \\
\hat{x}\left(k+N_{c} \mid k\right)
\end{array}\right]= \\
& =\left[\begin{array}{c}
A x(k)+B_{T} u(k) \\
A^{2} x(k)+\sum_{i=0}^{1} A^{1-i} B_{T} u(k+i) \\
\vdots \\
A^{N_{c}} x(k)+\sum_{i=0}^{N_{c}-1} A^{N_{c}-1-i} B_{T} u(k+i)
\end{array}\right]
\end{aligned}
$$

which can be expressed as

$$
\mathbf{x}=\mathbf{P} x(k)+\mathbf{H u}
$$

where $\mathbf{u}=\left[u(k)^{T} \ldots u\left(k+N_{c}-1\right)^{T}\right]^{T}$ is the vector of the $N_{c}$ future control actions, $\mathbf{P}$ and $\mathbf{H}$ are defined as

$\mathbf{P}=\left[\begin{array}{l}A \\ A^{2} \\ \vdots \\ A^{N_{c}}\end{array}\right], \mathbf{H}=\left[\begin{array}{cccc}B_{T} & 0 & \cdots & 0 \\ A B_{T} & B_{T} & \cdots & 0 \\ \vdots & \vdots & \ddots & \vdots \\ A^{N_{c}-1} B_{T} A^{N_{c}-2} B_{T} & \cdots & B_{T}\end{array}\right]$

At each step, the control action $u(k)$ is obtained by minimizing with respect to the sequence $\mathbf{u}$ of future control moves the following performance index

$$
J(k)=\mathbf{x}^{T} \mathbf{Q} \mathbf{x}+\mathbf{u}^{T} \mathbf{R} \mathbf{u}
$$

where $\mathbf{Q}=\operatorname{diag}(Q, \cdots, Q) \geq 0$ and $\mathbf{R}=$ $\operatorname{diag}(R, \cdots, R)>0$. The idea of the predictive approach to magnetic control is to impose the orthogonality between the geomagnetic field vector $b(k \Delta)$ and the control vector $T_{\text {mag }}(k)$ via the constraint

$$
b(k \Delta)^{T} u(k)=0
$$

which can be expressed in term of $\mathbf{u}$ as

$$
\mathbf{G u}=\left[b(k \Delta)^{T} 0 \ldots 0\right] \mathbf{u}=0
$$

By the use of Lagrange multipliers, the optimal solution of the constrained minimization problem defined by (15) and (16) is given by

$\mathbf{u}_{o p t}=-\Lambda\left(I-\mathbf{G}^{T}\left(\mathbf{G} \Lambda \mathbf{G}^{T}\right)^{-1} \mathbf{G} \Lambda\right) \mathbf{H}^{T} \mathbf{Q P} x(k)$ where $\Lambda=\left(\mathbf{H}^{T} \mathbf{Q H}+\mathbf{R}\right)^{-1}$. According to a receding horizon strategy, the above equation has to be evaluated at every sampling time, while only the first element of $\mathbf{u}_{o p t}$ is effectively used as control signal $u_{\text {opt }}(k)$. Since $\mathbf{G}$ depends on the periodic geomagnetic field vector $b(k \Delta)$, the optimal control $u_{\text {opt }}(k)$ can be interpreted as a periodic state-feedback controller. The corresponding vector of the coils' magnetic dipoles $m_{\text {opt }}(k)$ is obtained by solving the equations

$$
\left\{\begin{aligned}
u_{o p t}(k)^{T} u_{o p t}(k) & =\left[m_{o p t}(k) \times b(k \Delta)\right]^{T} u_{o p t}(k) \\
& =\left[b(k \Delta) \times u_{o p t}(k)\right]^{T} m_{o p t}(k) \\
u_{o p t}(k)^{T} m_{o p t}(k) & =0 \\
b(k \Delta)^{T} m_{o p t}(k) & =0
\end{aligned}\right.
$$

and can be computed as

$$
m_{\text {opt }}(k)=\frac{b(k \Delta) \times u_{\text {opt }}(k)}{|b(k \Delta)|_{2}^{2}} .
$$

The predictive approach proposed herein leads to a very simple solution in which the time dependence of the controller is carried by the measurable magnetic field vector $b(k \Delta)$, therefore, this controller is very easy to implement. The limitation of this simple formulation of the predictive approach, however, is that it does not guarantee closed-loop stability a priori.

\subsection{Fixed structure projection based controller}

A very common approach to the design of attitude controllers for magnetically actuated satellites of the form (10) is to consider control laws of the kind

$$
u(k)=m(k)=-S(b(k \Delta))^{T} K x(k) .
$$

The advantage of this controller structure is that only a fixed gain $K$ has to be designed, while the timedependence of the control law is carried by the (measurable) value of the geomagnetic field $b$. However, to the best knowledge of the authors, no suitable design approaches to the selection of gain $K$ in (17) are available.

In this paper, we propose to face this design problem using the approach to the solution of optimal periodic output feedback problems first presented in (Varga and Pieters, 1998). This approach relies on a gradient-based optimization approach to determine time-periodic output feedback controllers by minimising the quadratic cost function (13). Clearly, the proposed controller (17) is a particular case of the broader class of output feedback controllers. Given the fact 
that the time-varying portion of the controller is already fixed, one needs to optimise (13) only with respect to the constant parameters of matrix $K$.

The application of the results presented in (Varga and Pieters, 1998) to this problem requires a way of designing an initial stabilising gain, in order to reduce the numerical difficulties associated with open loop unstable dynamics, and to facilitate the convergence of the iterative optimization procedure. To this purpose, the initial gain of the controller has been selected according to the guidelines provided by (Lovera and Astolfi, 2004, Proposition 1) for the globally stabilising tuning of state feedback magnetic attitude controller of the "projection" type (i.e., equation (17)).

¿From a numerical point of view, the optimal periodic output-feedback gain $K$ in the control law (17) which minimizes the performance index (13) can be computed using a suitable function available in the Periodic Systems Toolbox (Varga, 2005b). This function is based on a gradient-based function minimization technique for problems with simple bounds (limited memory BFGS). To achieve the highest efficiency, the function and gradient evaluations have been implemented as a Fortran 95 mex-function based on the formulas derived in (Varga and Pieters, 1998).

\section{SIMULATION RESULTS}

The considered spacecraft is of the type described in Section 2 and operates in a near polar ( $87^{\circ}$ inclination) orbit with an altitude of $450 \mathrm{~km}$ and a corresponding orbit period of about $5600 \mathrm{~s}$. The numerical values of parameters used in the mathematical model are:

- Satellite inertia: $I=\operatorname{diag}\left[\begin{array}{lll}35 & 16 & 25\end{array}\right]$;

- Momentum wheel inertia $\left(\mathrm{kgm}^{2}\right): J=0.01$;

- Orbital angular rate $(\mathrm{rad} / \mathrm{s}): \Omega_{0}=0.001194$;

- Nominal wheel speed ( $\mathrm{rad} / \mathrm{s}): \bar{\Omega}=200$;

- Nominal (periodic) magnetic field components (Tesla), used for design purposes only:

$$
b(t)=10^{-6}\left[\begin{array}{c}
7 \cos \left(\Omega_{0} t\right)+48 \sin \left(\Omega_{0} t\right) \\
23 \cos \left(\Omega_{0} t\right)-2 \sin \left(\Omega_{0} t\right) \\
5
\end{array}\right] .
$$

Magnetic coils with a saturation limit of $\pm 20 \mathrm{Am}^{2}$ have been considered.

For this satellite, a discrete-time model of the form (11)-(12) has been computed, by choosing a discretetime period $\mathrm{N}=500$ (which corresponds to a sampling interval $\Delta$ of about 11.3 seconds) and three controllers have been designed, using the optimal LQ approach, the predictive approach and the fixed structure projection approach. In all three cases, the weighting matrices in the corresponding quadratic cost functions have been chosen equal to $Q=0.01 I_{6}$ and $R=100 I_{3}$. The results obtained from the three designs can be summarised as follows:
- Closed loop stability: as can be seen from Table 1 , all the designed compensators lead to asymptotically stable closed loop dynamics. However, as mentioned previously, stability in the case of the predictive controller is not guaranteed but must be verified a posteriori. The value of control horizon $N_{c}$ used for computations was 30 .

- Optimality: the results obtained using periodic optimal control and the fixed structure controller can be compared directly in terms of the achieved optimal value of the cost function (13). In particular, it is interesting to note that the performance loss associated with the adoption of the fixed structure controller instead of the optimal periodic one turns out to be limited to about $20 \%$ (the optimal function value is 228.52 for the optimal controller and 273.47 for the fixed structure one) which can be definitely considered acceptable.

Finally, in order to illustrate the time domain behavior of the fixed structure controller, a typical simulation example is presented, which shows the transients following a (small) initial perturbation of the attitude dynamics with respect to the nominal Earth pointing equilibrium. The simulations have been carried out using the tools presented in (Annoni et al., 1999; Lovera, 2003), on the basis of the models for the space environment described in the classical references (Sidi, 1997; Wertz, 1978). As can be seen from Figure 1 the proposed controller provides closed loop transients of about half an orbit and a very good level of performance in terms of attenuation of external disturbance torques.

\section{CONCLUDING REMARKS}

The attitude control problem for a small spacecraft using magnetic actuators has been considered and analyzed. In particular, a novel approach to the design of "projection based" magnetic attitude controllers has been proposed, which is based on the solution of a periodic LQ-optimal output feedback control problem with constant feedback. The performance of this approach compared with that achievable with a periodic LQ-optimal state feedback controller is very satisfactory (nearly optimal). Future work will aim at generalising the proposed approach to output feedback and disturbance attenuation schemes.

\section{ACKNOWLEDGMENTS}

Paper supported by the Italian National Research Project "New Techniques of Identification and Adaptive Control for Industrial Systems".

\section{REFERENCES}

Annoni, G., E. De Marchi, F. Diani, M. Lovera and G.D. Morea (1999). Standardising tools for attitude control system design: the MITA platform 
Table 1. Open and closed loop characteristic multipliers

\begin{tabular}{cccc}
\hline Open loop & Optimal state-feedback control & Predictive control & Fixed structure controller \\
\hline $9.9864 \mathrm{e}-001+5.2216 \mathrm{e}-002 \mathrm{i}$ & $7.4635 \mathrm{e}-001+3.8905 \mathrm{e}-002 \mathrm{ii}$ & $-6.0064 \mathrm{e}-002+2.1589 \mathrm{e}-002 \mathrm{i}$ & $2.6381 \mathrm{e}-003$ \\
$9.9864 \mathrm{e}-001-5.2216 \mathrm{e}-002 \mathrm{i}$ & $7.4635 \mathrm{e}-001-3.8905 \mathrm{e}-002 \mathrm{i}$ & $-6.0064 \mathrm{e}-002-2.1589 \mathrm{e}-002 \mathrm{i}$ & $3.0363 \mathrm{e}-003+1.5439 \mathrm{e}-003 \mathrm{i}$ \\
$2.1148 \mathrm{e}-001+9.7745 \mathrm{e}-001 \mathrm{i}$ & $1.5543 \mathrm{e}-001+7.1907 \mathrm{e}-001 \mathrm{i}$ & $3.9234 \mathrm{e}-003+3.4159 \mathrm{e}-002 \mathrm{i}$ & $3.0363 \mathrm{e}-003-1.5439 \mathrm{e}-003 \mathrm{i}$ \\
$2.1148 \mathrm{e}-001-9.7745 \mathrm{e}-001 \mathrm{i}$ & $1.5543 \mathrm{e}-001-7.1907 \mathrm{e}-001 \mathrm{i}$ & $3.9234 \mathrm{e}-003-3.4159 \mathrm{e}-002 \mathrm{i}$ & $3.7574 \mathrm{e}-004$ \\
$1.0243 \mathrm{e}+004$ & $5.7661 \mathrm{e}-006+3.4588 \mathrm{e}-006 \mathrm{i}$ & $-8.0621 \mathrm{e}-013+1.1602 \mathrm{e}-012 \mathrm{i}$ & $1.8609 \mathrm{e}-026+1.3216 \mathrm{e}-026 \mathrm{i}$ \\
$9.7627 \mathrm{e}-005$ & $5.7661 \mathrm{e}-006-3.4588 \mathrm{e}-006 \mathrm{i}$ & $-8.0621 \mathrm{e}-013-1.1602 \mathrm{e}-012 \mathrm{i}$ & $1.8609 \mathrm{e}-026-1.3216 \mathrm{e}-026 \mathrm{i}$ \\
\hline
\end{tabular}
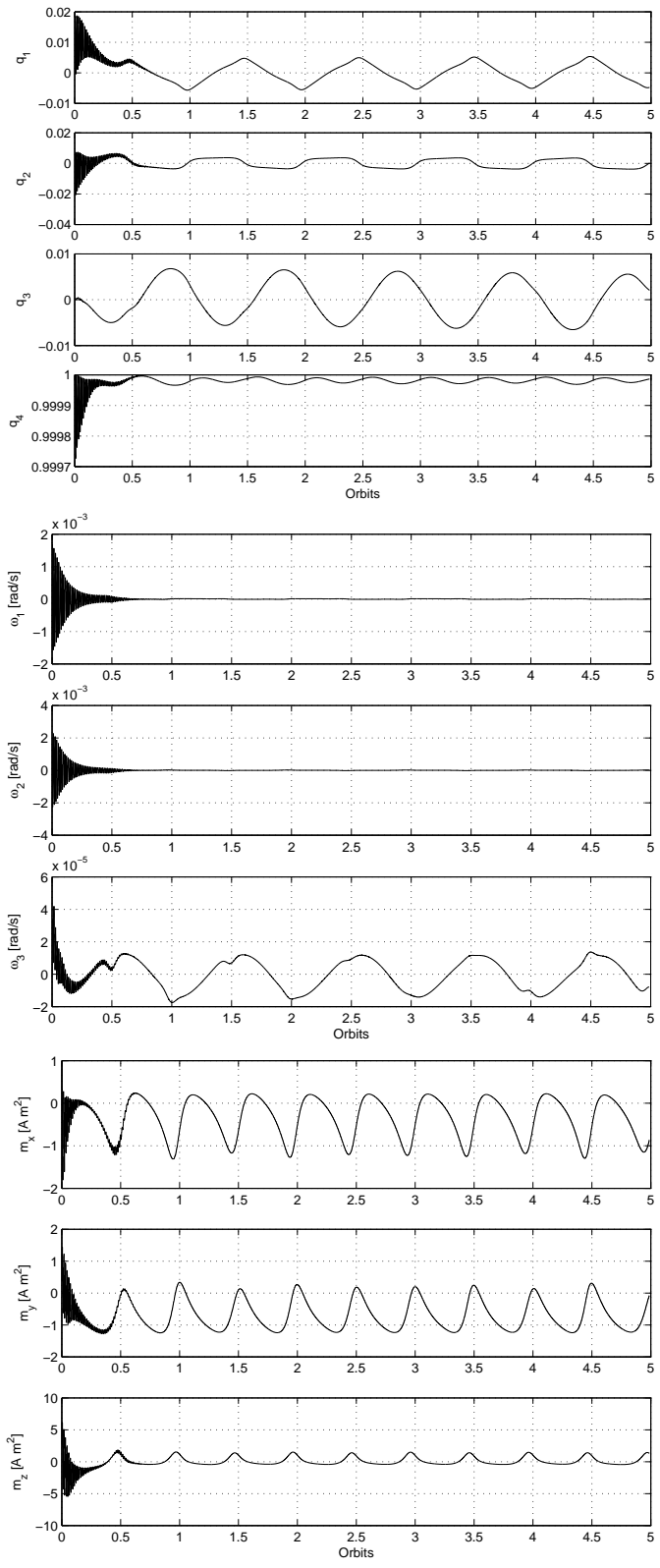

Fig. 1. Quaternion, angular rates and control dipole moments: output feedback controller.

experience. Data Systems in Aerospace (DASIA) 1999, Lisbon, Portugal.

Arduini, C. and P. Baiocco (1997). Active magnetic damping attitude control for gravity gradient stabilised spacecraft. Journal of Guidance and Control 20(1), 117-122.

Camacho, E. F. and C. Bordons (1998). Model Predictive Control. Springer-Verlag. Berlin.

Hughes, P. (1986). Spacecraft attitude dynamics. John Wiley and Sons.
Lovera, M. (2001). Optimal magnetic momentum control for inertially pointing spacecraft. European Journal of Control 7(1), 30-39.

Lovera, M. (2003). Modelling and simulation of spacecraft attitude dynamics. Proc. of 4th International Symposium on Mathematical Modelling, Vienna, Austria.

Lovera, M. and A. Astolfi (2004). Spacecraft attitude control using magnetic actuators. Automatica 40(8), 1405-1414.

Lovera, M., E. De Marchi and S. Bittanti (2002). Periodic attitude control techniques for small satellites with magnetic actuators. IEEE Transactions on Control Systems Technology 10(1), 90-95.

Pittelkau, M. (1993). Optimal periodic control for spacecraft pointing and attitude determination. Journal of Guidance, Control and Dynamics 16(6), 1078-1084.

Psiaki, M. (2001). Magnetic torquer attitude control via asymptotic periodic linear quadratic regulation. Journal of Guidance, Control and Dynamics 24(2), 386-394.

Sidi, M. (1997). Spacecraft dynamics and control. Cambridge University Press.

Silani, E. and M. Lovera (2005). Magnetic spacecraft attitude control: A survey and some new results. Control Engineering Practice 13(3), 357-371.

Varga, A. (2005a). On solving discrete-time periodic Riccati equations. Prep. of 16th IFAC World Congress, Prague, Czech Republic.

Varga, A. (2005b). A PERIOdIC Systems Toolbox for MATLAB. Prepr. of 16th IFAC World Congress, Prague, Czech Republic.

Varga, A. and S. Pieters (1998). Gradient-based approach to solve optimal periodic output feedback control problems. Automatica 34, 477-481.

Wertz, J. (1978). Spacecraft attitude determination and control. D. Reidel Publishing Company.

Wisniewski, R. and J. Stoustrup (2002). Periodic $H_{2}$ synthesis for spacecraft attitude determination and control with a vector magnetometer and magnetorquers. Proc. of 1st IFAC Workshop on Periodic Control Systems, Cernobbio-Como, Italy, pp. 121-126.

Wisniewski, R. and L.M. Markley (1999). Optimal magnetic attitude control. Proc. of 14th IFAC World Congress, Beijing, China.

Wisniewski, R. and M. Blanke (1999). Fully magnetic attitude control for spacecraft subject to gravity gradient. Automatica 35(7), 1201-1214. 